\title{
Exploring the Association Between Prostate Cancer Diagnosis per se and use of GnRH Agonists and Diabetes Control in Men with type 2 Diabetes Mellitus: A Nationwide, Population-Based Cohort Study
}

E Lin ( $\nabla$ e.lin@kcl.ac.uk)

King's College London

Hans Garmo

King's College London

Mieke Hemelrijck

King's College London

Jan Adolfsson

Karolinska Institute

Pär Stattin

Uppsala University

Björn Zethelius

Uppsala University

Danielle Crawley

King's College London

\section{Research Article}

Keywords: Prostate cancer diagnosis, GnRH agonists, diabetes

Posted Date: July 14th, 2021

DOI: https://doi.org/10.21203/rs.3.rs-599963/v1

License: (c) (1) This work is licensed under a Creative Commons Attribution 4.0 International License.

Read Full License 


\section{Abstract}

Background: Gonadotropin Releasing Hormones agonists ( $\mathrm{GnRH})$, which are first line treatment for metastatic prostate cancer (PCa), increase risk of type 2 diabetes mellitus (T2DM). This study aims to quantify the association of use of $\mathrm{GnRH}$ with diabetes control in PCa men with T2DM.

Methods: Nationwide population-based cohort study in the Swedish National Diabetes Register and Prostate Cancer data Base Sweden 4.1, on the association between GnRH and diabetes control in T2DM men with PCa by comparing T2DM men with PCa vs. without PCa, as well as comparing T2DM men with $\mathrm{PCa}$ on or not on $\mathrm{GnRH}$. The primary exposure was use of $\mathrm{GnRH}$. Worsening diabetes control was the primary outcome, defined as: 1) increase of $\mathrm{HbA} 1 \mathrm{c}$ to $58 \mathrm{mmol} / \mathrm{mol}$ or higher; 2) $\mathrm{HbA} 1 \mathrm{c}$ increase by 10 $\mathrm{mmol} / \mathrm{mol}$ or more; 3) Start of antidiabetic drugs or switch to insulin; 4) combine all definitions above. Cox proportional hazards regression was used to analyze the association.

Results: There were 5,714 T2DM men with PCa of whom 692 were on GnRH and 28,445 PCa-free men with T2DM with similar baseline characteristics. Diabetes control was worse in men with GnRH vs. PCafree men (HR: 1.24, 95\% Cl: 1.13-1.34) as well as compared with PCa men without GnRH (HR:1.58, 95\% Cl: 1.39-1.80).

Conclusion: Use of GnRH in T2DM men with PCa was associated with worse glycemic control. The findings highlight the need to closely monitor diabetes control in men with T2DM and PCa starting GnRH agonists and to limit the duration of their use when possible.

\section{Background}

Prostate cancer ( $\mathrm{PCa}$ ) is the most frequently diagnosed cancer in men in Europe, with approximately 450, 000 new cases in 2018 , accounting for $24 \%$ of all newly diagnosed cancers [1]. While, in 2019, about 59 million people in Europe had a diagnosis of type two diabetes (T2DM) [2]. Thus, PCa and T2DM are common conditions that may occur concurrently in the same man [3]. Few studies have assessed the association of PCa and its hormonal treatment with diabetes control in men with pre-existing T2DM.

Gonadotropin-releasing hormone agonists $(\mathrm{GnRH})$ are first line treatment for metastatic PCa and are also widely used in conjunction with radiotherapy in locally advanced PCa as both neoadjuvant and adjuvant therapy [4]. GnRH have a range of side effects, including a metabolic like syndrome [5]. An association between use of $\mathrm{GnRH}$ and T2DM has been demonstrated in many observational studies, and it is established that $\mathrm{GnRH}$ agonists lead to increased insulin resistance and risk of diabetes [6-8]. In 2010, the Food and Drug Administration required labelling of all GnRH with a warning of an increased risk of diabetes and cardiovascular diseases [9]. However, few studies have examined the effect of GnRH on diabetes control in men with pre-existing T2DM.

Our aim was to investigate the association between use of $\mathrm{GnRH}$ and diabetes control, both in terms of glycemic control and changes in antidiabetic drugs, in men with T2DM and PCa. 


\section{Methods}

\section{Data source}

Prostate Cancer data Base Sweden (PCBaSe) 4.1 is a database based on the National Prostate Cancer Register (NPCR) of Sweden, which contains information on $98 \%$ of men in Sweden diagnosed with PCa between 1998 and 2016 compared with The Cancer Registry to which reporting is mandated [10]. In PCBaSe 4.1, men in NPCR were linked to other nationwide databases, including National Patient Register, Longitudinal integrated database for health insurance and labor market studies, Swedish National Cancer Register and other nationwide registers [10] by use of the unique personal identity number of all residents. We obtained data on PCa characteristics, co-morbidities, civil status and educational level from PCBaSe 4.1 [10]. We also collected prescribed medications data from the National Prescribed Drug Register (PDR) which was established in July 2005 [11]. The PDR contains information of all prescribed drugs dispensed at pharmacies covering the whole population of Sweden.

Moreover, information on diabetic conditions was retrieved through a linkage between PCBaSe 4.1 and the National Diabetes Registry (NDR) which was initiated in 1996 and has engaged the participation of both hospitals and primary care. This register contains detailed data on demographics, smoking, diabetes duration, treatment modalities, risk factors and diabetes complications and it currently includes most of T2DM patients age 18 and older in Sweden $[12,13]$.

The study population included men diagnosed with T2DM, according to NDR, amongst men included in PCBaSe 4.1 between 2006 and 2016.

\section{Study population}

To investigate the association of use of $\mathrm{GnRH}$ and a PCa diagnosis per se with diabetes control separately, we created two cohorts of men with a diagnosis of T2DM - "PCa cohort" and "PCa + GnRH cohort" (Fig. 1). In the PCa cohort, we included men with at least four registrations of data in NDR who were diagnosed with PCa after their third NDR-registration. Date of PCa diagnosis was considered as start of follow up. For each man with PCa in our study, five PCa-free men with T2DM were randomly selected from the NDR, matched on average duration between NDR visits and number of previous NDR registrations. Start of follow-up for these men was 'inherited' from the corresponding man with a PCa diagnosis.

The PCa $+\mathrm{GnRH}$ cohort consisted of men with PCa and T2DM who initiated use of GnRH after the third registered date in the NDR. Date of the first filled prescription for GnRH registered in the PDR was considered start of follow up. As a comparison, we selected five men with PCa not on GnRH randomly from the NDR, matched on average duration between NDR visits and the number of previous NDR registrations. Start of follow up for these men was 'inherited' from the corresponding man treated with $\mathrm{GnRH}$.

\section{Exposures}


The primary exposure was PCa diagnosis. In addition, we also used information on PCa risk categories and use of GnRH from NPCR and PDR. According to the National Comprehensive Cancer Network (NCCN) guideline, there are five risk categories for PCa: 1) low-risk category: T1 or T2a stage, PSA $<10 \mathrm{ng} / \mathrm{mL}$ and Gleason score 6; ii) intermediate-risk category: T2b or T2c stage, $10 \mathrm{ng} / \mathrm{mL}, \mathrm{PSA}<20 \mathrm{ng} / \mathrm{mL}$, or Gleason score 7; iii) high-risk category: T3a or T4 stage, $\mathrm{PSA} \geq 20 \mathrm{ng} / \mathrm{mL}$, or Gleason score $\geq 8$; iv) regional metastases category: any T, N1 and M0 stage; $\mathrm{v}$ ) distant metastases category: any T or $\mathrm{N}$ and $\mathrm{M} 1$ stage[14].

\section{Outcomes}

The primary outcome was worsening of diabetes control based on information collected as part of the longitudinal follow-up in the NDR. According to NICE guidance, the definition of worsening diabetes control includes [15]:

1. $\mathrm{HbA} 1 \mathrm{c}$ rose to $58 \mathrm{mmol} / \mathrm{mol}$ or higher (for men not already $>58 \mathrm{mmol} / \mathrm{mol}$ at baseline)

2. HbA1c was $10 \mathrm{mmol} / \mathrm{mol}$ higher than the baseline measurement.

3. Commencement of antidiabetic drugs or switch to insulin (for men not already on insulin at baseline).

We also combined the above criteria. For men whose $\mathrm{HbA} 1 \mathrm{c}$ was less than $58 \mathrm{mmol} / \mathrm{L}$ and who did not use insulin at the baseline, we included all of the above definitions with whichever occurred first as the combination event. For men whose $\mathrm{HbA} 1 \mathrm{c}$ was over $58 \mathrm{mmol} / \mathrm{L}$ and/or who used insulin the baseline, we used the remaining definitions, with whichever came first as the combination event.

\section{Data analysis}

We used registrations in the period 1/1 2006 to 31/12 2017. The baseline measurements for a participant were based on the three last NDR-registrations prior to the start of follow up. Missing data was imputed using last observation carried forward, i.e., if the last observation in NDR was missing then information was taken from the second last, if that was also missing it was retrieved from the third last NDR registration. If all the last three NDR observations were missing, then data was classified as missing.

Time to event was defined as the time from start of follow up to the first date of worsening diabetes control or last observation in NDR, whichever came first. Hazard ratios (HR) and 95\% confidence interval (Cl) for worsening of diabetes control as defined above were obtained using Cox proportional hazards regression models. All models were adjusted for age at PCa onset, duration of T2DM, education level, civil status, the Charlson Comorbidity Index (CCl), smoking habits, physical activity and body mass index (BMI). Cumulative incidence of worsening T2DM control was presented using Kaplan-Meier curves.

All data management was performed with Statistical Analysis Systems release 9.4 (SAS Institute, Cary, NC) and R 3.5.2 (R Foundation for Statistical Computing). The study has been approved by The Research Ethics Board at Uppsala University, Sweden. 


\section{Results}

5,714 men with PCa and 28,445 PCa-free men were included in the PCa cohort. The PCa + GnRH cohort contained 692 PCa men who started GnRH after PCa diagnosis and 3,460 PCa men not using GnRH as comparison. Baseline characteristic for age, education level, civil status, $\mathrm{CCl}$, smoking habits, $\mathrm{BMI}$, physical activity, and T2DM status (including T2DM duration, T2DM treatments and HbA1c) were similar between PCa men or PCa men with GnRH and the comparison groups in both PCa cohort and PCa + GnRH cohort, respectively (Table 1 ). 
Table 1

Baseline characteristics of men in NDR diagnosed with prostate cancer and/or used GnRH agonists between 2006 and 2016 and their matched comparison

PCa cohort

$\begin{array}{ll}\text { PCa men } & \text { No PCa } \\ (\mathrm{N}= & \text { men }(\mathrm{N}= \\ 5,714) & 28,445)\end{array}$

$\mathrm{PCa}+\mathrm{GnRH}$ cohort

PCa using

GnRH men (N

=692)
PCa without using

GnRH men ( $\mathrm{N}=$ 3,460 )

Patients characteristics

Age (year), №. (\%)

$<60$

60-69

$70-79$

$80+$

Education level, No. (\%)

Low

Middle

High

Missing

Civil status, No. (\%)

Married

3,708

(64.9)

2,755
$(48.2)$

570

(10.0)

$49(0.9)$
12,231

(43.0)

13,172

(46.3)

2,686 (9.4)

$356(1.3)$

$5(0.7)$

297 (42.9)

$82(11.8)$

$432(12.5)$

$17(0.5)$

$2,006 \quad 10,863$

(35.1)

(38.2)

17,582

(61.8)

Not married $(+$

Divorced/Widower/missing)

$\mathrm{CCl}$, No. (\%)

0

1

\begin{tabular}{llll}
2,413 & $\begin{array}{l}10,319 \\
(36.3)\end{array}$ & $197(28.5)$ & $942(27.2)$ \\
\hline$(42.2)$ & & \\
\hline 1,348 & $6,494(22.8)$ & $174(25.1)$ & $1,014(29.3)$ \\
$(23.6)$ & & & \\
\hline
\end{tabular}

PCa denotes Prostate Cancer, T2DM; Type 2 diabetes mellitus, BMl; body mass index, CCl; Charlson Comorbidity Index 


\begin{tabular}{|c|c|c|c|c|}
\hline \multirow[b]{2}{*}{2} & \multicolumn{2}{|c|}{ PCa cohort } & \multicolumn{2}{|c|}{$\mathrm{PCa}+\mathrm{GnRH}$ cohort } \\
\hline & $\begin{array}{l}765 \\
(13.4)\end{array}$ & $4,246(14.9)$ & 109 (15.8) & $538(15.5)$ \\
\hline $3+$ & $\begin{array}{l}1,188 \\
(20.8)\end{array}$ & $7,386(26.0)$ & $212(30.6)$ & $966(27.9)$ \\
\hline \multicolumn{5}{|l|}{ Smoking, No. (\%) } \\
\hline No & $\begin{array}{l}4,581 \\
(80.2)\end{array}$ & $\begin{array}{l}22,273 \\
(78.3)\end{array}$ & $524(75.7)$ & $2,683(77.5)$ \\
\hline Yes & $553(9.7)$ & $2,912(10.2)$ & $51(7.4)$ & $267(7.7)$ \\
\hline Missing & $\begin{array}{l}580 \\
(10.2)\end{array}$ & $3,260(11.5)$ & $117(16.9)$ & $510(14.7)$ \\
\hline \multicolumn{5}{|c|}{$\begin{array}{l}\text { Times of at least } 60 \text { minutes } \\
\text { physical activity in } 7 \text { days, No. } \\
\text { (\%) }\end{array}$} \\
\hline Daily & $\begin{array}{l}573 \\
(10.0)\end{array}$ & $3,452(12.1)$ & $98(14.2)$ & $380(11.0)$ \\
\hline $3-5$ times a week & $500(8.8)$ & $2,486(8.7)$ & $69(10.0)$ & $268(7.7)$ \\
\hline 1-2 times a week & $\begin{array}{l}902 \\
(15.8)\end{array}$ & $4,261(15.0)$ & $96(13.9)$ & $503(14.5)$ \\
\hline Less than once a week & $\begin{array}{l}1,016 \\
(17.8)\end{array}$ & $4,786(16.8)$ & $104(15.0)$ & $629(18.2)$ \\
\hline Never & $\begin{array}{l}1,531 \\
(26.8)\end{array}$ & $6,919(24.3)$ & $140(20.2)$ & $784(22.7)$ \\
\hline Missing & $\begin{array}{l}1,192 \\
(20.9)\end{array}$ & $6,541(23.0)$ & $185(26.7)$ & $896(25.9)$ \\
\hline \multicolumn{5}{|l|}{ BMI (kg/m2), No. (\%) } \\
\hline$<25$ & $\begin{array}{l}969 \\
(17.0)\end{array}$ & 4,668 (16.4) & $111(16.0)$ & $610(17.6)$ \\
\hline $25-29$ & $\begin{array}{l}2,509 \\
(43.9)\end{array}$ & $\begin{array}{l}11,845 \\
(41.6)\end{array}$ & $291(42.1)$ & $1,507(43.6)$ \\
\hline $30-34$ & $\begin{array}{l}1,342 \\
(23.5)\end{array}$ & 6,677 (23.5) & $150(21.7)$ & $731(21.1)$ \\
\hline $35-39$ & $353(6.2)$ & $1,961(6.9)$ & $39(5.6)$ & $187(5.4)$ \\
\hline $40+$ & $91(1.6)$ & $612(2.2)$ & $15(2.2)$ & $52(1.5)$ \\
\hline
\end{tabular}

PCa denotes Prostate Cancer, T2DM; Type 2 diabetes mellitus, BMl; body mass index, CCl; Charlson Comorbidity Index 


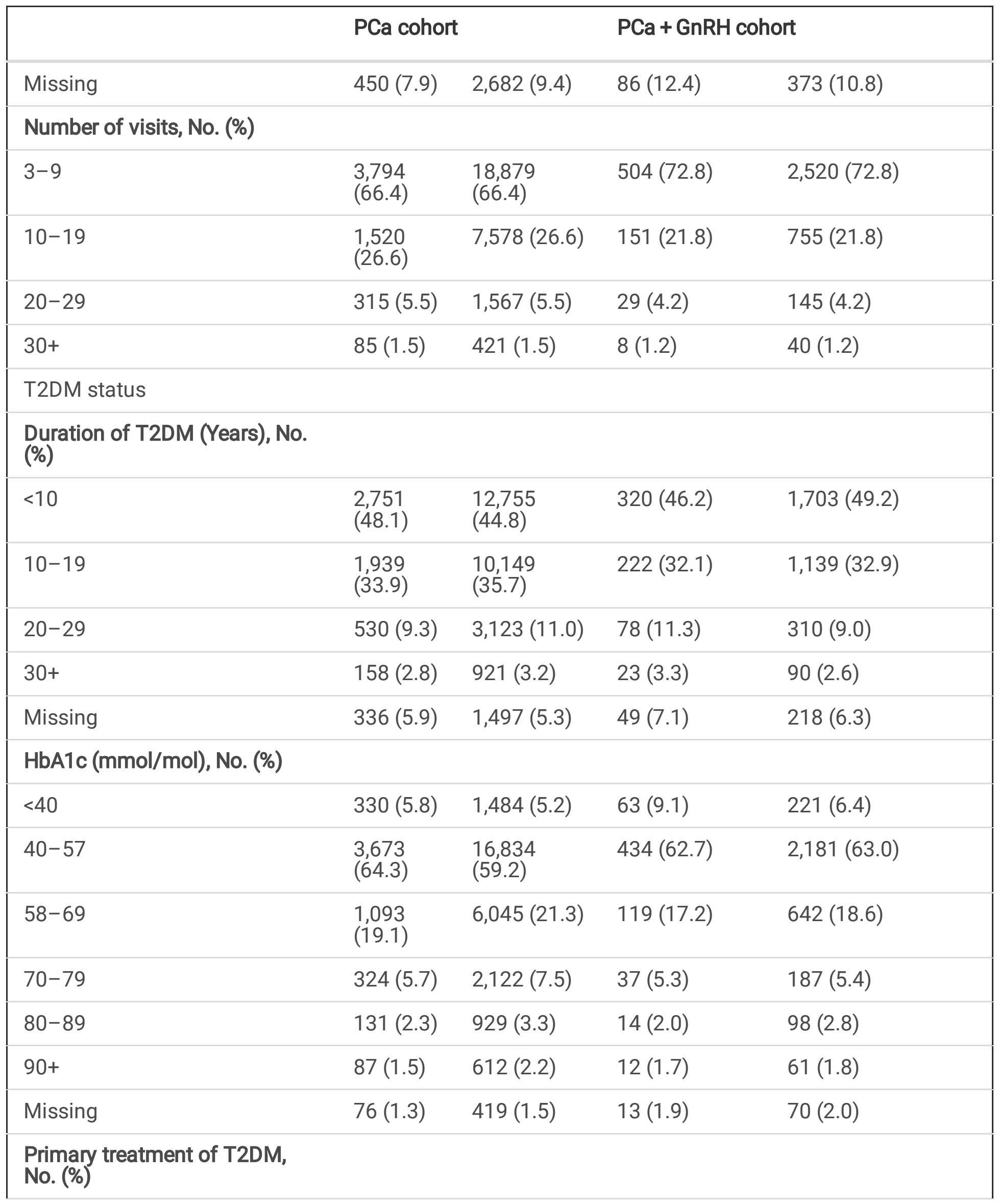

PCa denotes Prostate Cancer, T2DM; Type 2 diabetes mellitus, BMl; body mass index, CCl; Charlson Comorbidity Index 


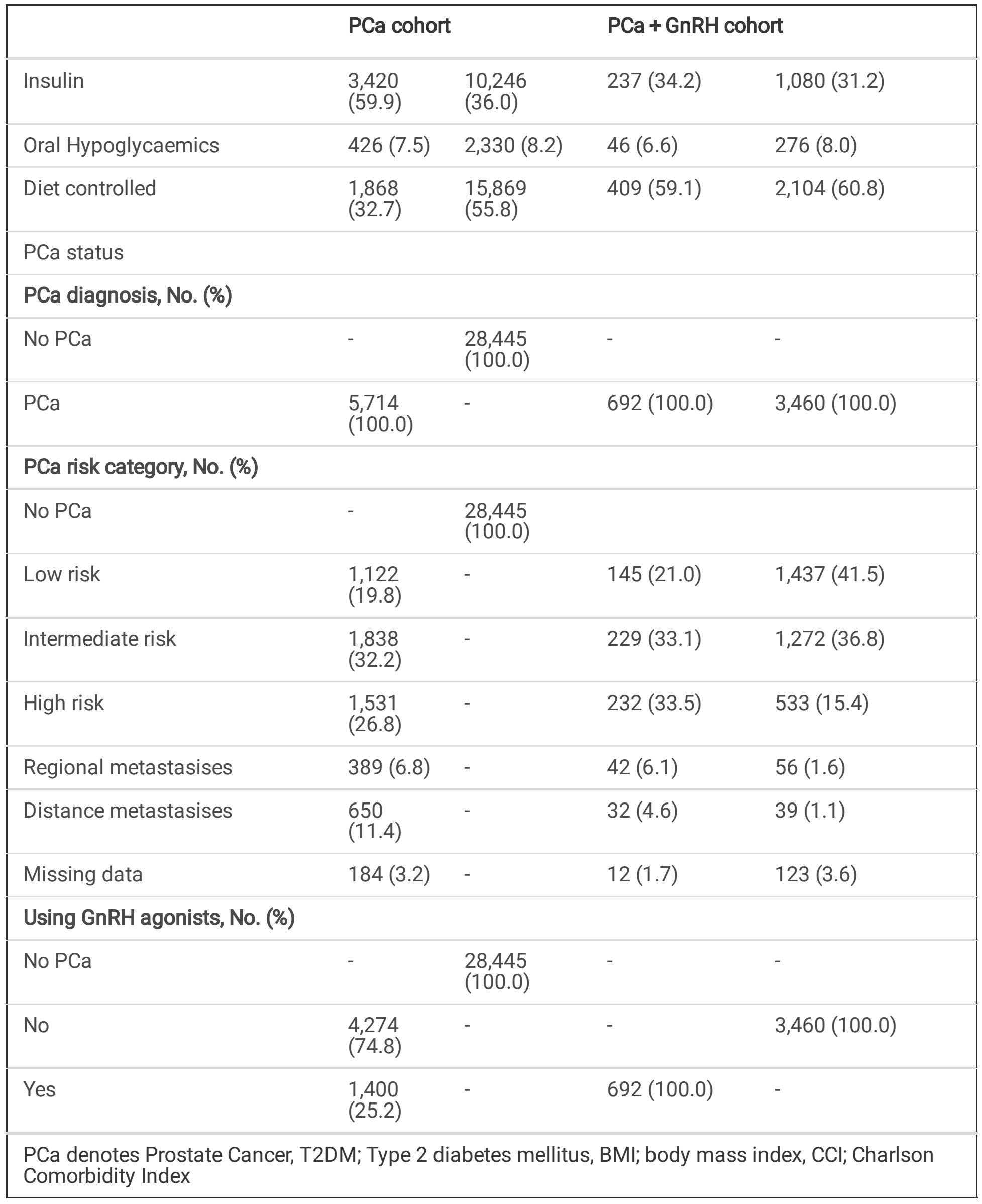

\section{PCa Cohort}


No association of PCa diagnosis (all risk categories combined) with diabetes control was found, compared to PCa-free men with T2DM (HR:1.04, 95\%Cl: 0.99-1.08) (Table 2). However, the risk of worsening diabetes control was increased in men with high-risk PCa. The HR for worsened diabetes control was 1.28 (95\% Cl: 1.10-1.50) for men with regional metastatic PCa, and 1.23 (95\% Cl: 1.09-1.40) in men with distant metastases, as compared with PCa-free men. Similar results were seen for the association between use of GnRH agonists and diabetes control (HR: 1.24, 95\% Cl: 1.13-1.34), compared to men without PCa, but no increased risk was seen for those with PCa not using GnRH agonists (HR: 0.98, 95\% Cl: 0.93-1.03). 
Table 2

$\mathrm{HR}$ and $95 \% \mathrm{Cl}$ for change in diabetes control in $\mathrm{PCa}$ cohort

\begin{tabular}{|c|c|c|c|c|c|c|c|c|}
\hline & \multicolumn{2}{|c|}{$\begin{array}{l}\mathrm{HbA1c} \text { equal to } \\
58 \mathrm{mmol} / \mathrm{mol}^{\mathrm{a}}\end{array}$} & \multicolumn{2}{|c|}{$\begin{array}{l}\text { HbA1c increased } \\
10 \mathrm{mmol} / \mathrm{mol}\end{array}$} & \multicolumn{2}{|c|}{$\begin{array}{l}\text { Change of } \\
\text { T2DM drugs } b\end{array}$} & \multicolumn{2}{|c|}{$\begin{array}{l}\text { Combination of } \\
\text { all definitions }\end{array}$} \\
\hline & HR & $95 \% \mathrm{Cl}$ & HR & $95 \% \mathrm{Cl}$ & HR & $95 \% \mathrm{Cl}$ & HR & $95 \% \mathrm{Cl}$ \\
\hline \multicolumn{9}{|l|}{ Crude model } \\
\hline \multicolumn{9}{|l|}{ PCa diagnosis } \\
\hline No & 1.00 & Ref. & 1.00 & ref. & 1.00 & ref. & 1.00 & ref. \\
\hline Yes & 0.99 & $\begin{array}{l}(0.94- \\
1.05)\end{array}$ & 1.10 & $\begin{array}{l}(1.04- \\
1.15)\end{array}$ & 0.98 & $\begin{array}{l}(0.89- \\
1.07)\end{array}$ & 1.02 & $\begin{array}{l}(0.97- \\
1.06)\end{array}$ \\
\hline \multicolumn{9}{|l|}{$\begin{array}{l}\text { PCa risk } \\
\text { category }\end{array}$} \\
\hline No PCa & 1.00 & ref. & 1.00 & ref. & 1.00 & ref. & 1.00 & ref. \\
\hline Low risk & 0.91 & $\begin{array}{l}(0.81- \\
1.02)\end{array}$ & 0.99 & $\begin{array}{l}(0.89- \\
1.10)\end{array}$ & 1.15 & $\begin{array}{l}(0.98- \\
1.36)\end{array}$ & 0.97 & $\begin{array}{l}(0.88- \\
1.06)\end{array}$ \\
\hline Intermediate risk & 0.97 & $\begin{array}{l}(0.89- \\
1.06)\end{array}$ & 1.00 & $\begin{array}{l}(0.92- \\
1.09)\end{array}$ & 0.94 & $\begin{array}{l}(0.81- \\
1.10)\end{array}$ & 1.00 & $\begin{array}{l}(0.93- \\
1.07)\end{array}$ \\
\hline High risk & 0.95 & $\begin{array}{l}(0.85- \\
1.05)\end{array}$ & 1.10 & $\begin{array}{l}(1.01- \\
1.21)\end{array}$ & 0.85 & $\begin{array}{l}(0.71- \\
1.01)\end{array}$ & 0.98 & $\begin{array}{l}(0.90- \\
1.06)\end{array}$ \\
\hline $\begin{array}{l}\text { Regional } \\
\text { metastasises }\end{array}$ & 1.25 & $\begin{array}{l}(1.04- \\
1.52)\end{array}$ & 1.44 & $\begin{array}{l}(1.21- \\
1.70)\end{array}$ & 1.06 & $\begin{array}{l}(0.76- \\
1.49)\end{array}$ & 1.25 & $\begin{array}{l}(1.07- \\
1.46)\end{array}$ \\
\hline $\begin{array}{l}\text { Distance } \\
\text { metastasises }\end{array}$ & 1.31 & $\begin{array}{l}(1.11- \\
1.53)\end{array}$ & 1.55 & $\begin{array}{l}(1.35- \\
1.78)\end{array}$ & 0.81 & $\begin{array}{l}(0.58- \\
1.13)\end{array}$ & 1.21 & $\begin{array}{l}(1.06- \\
1.38)\end{array}$ \\
\hline Missing data & 1.04 & $\begin{array}{l}(0.77- \\
1.40)\end{array}$ & 1.06 & $\begin{array}{l}(0.82- \\
1.38)\end{array}$ & 1.34 & $\begin{array}{l}(0.87- \\
2.06)\end{array}$ & 0.95 & $\begin{array}{l}(0.75- \\
1.20)\end{array}$ \\
\hline \multicolumn{9}{|l|}{$\begin{array}{l}\text { Using GnRH } \\
\text { agonists, n (\%) }\end{array}$} \\
\hline No PCa & 1.00 & ref. & 1.00 & ref. & 1.00 & ref. & 1.00 & ref. \\
\hline No & 0.94 & $(0.88-1.00)$ & 1.02 & $\begin{array}{l}(0.96- \\
1.08)\end{array}$ & 0.94 & $\begin{array}{l}(0.84- \\
1.04)\end{array}$ & 0.96 & $\begin{array}{l}(0.92- \\
1.01)\end{array}$ \\
\hline Yes & 1.20 & $\begin{array}{l}(1.08- \\
1.33)\end{array}$ & 1.38 & $\begin{array}{l}(1.26- \\
1.51)\end{array}$ & 1.13 & $\begin{array}{l}(0.95- \\
1.34)\end{array}$ & 1.21 & $\begin{array}{l}(1.11- \\
1.31)\end{array}$ \\
\hline
\end{tabular}

a. Men with a HbA1c over $58 \mathrm{mmol} / \mathrm{I}$ at baseline excluded

b. Men using insulin at the baseline excluded

c. This model was adjusted for age at PCa diagnosis, duration of T2DM, education level, $\mathrm{CCl}$, civil status, smoking habits, physical activity and BMI. 


\begin{tabular}{|c|c|c|c|c|c|c|c|c|}
\hline & \multicolumn{2}{|c|}{$\begin{array}{l}\text { HbA1c equal to } \\
58 \mathrm{mmol} / \mathrm{mol}^{\mathrm{a}}\end{array}$} & \multicolumn{2}{|c|}{$\begin{array}{l}\text { HbA1c increased } \\
10 \mathrm{mmol} / \mathrm{mol}\end{array}$} & \multicolumn{2}{|c|}{$\begin{array}{l}\text { Change of } \\
\text { T2DM drugs }\end{array}$} & \multicolumn{2}{|c|}{$\begin{array}{l}\text { Combination of } \\
\text { all definitions }\end{array}$} \\
\hline & HR & $95 \% \mathrm{Cl}$ & HR & $95 \% \mathrm{Cl}$ & HR & $95 \% \mathrm{Cl}$ & HR & $95 \% \mathrm{Cl}$ \\
\hline \multicolumn{9}{|l|}{$\begin{array}{l}\text { Adjusted model } \\
\text { c }\end{array}$} \\
\hline \multicolumn{9}{|l|}{ PCa diagnosis } \\
\hline No & 1.00 & ref. & 1.00 & ref. & 1.00 & ref. & 1.00 & ref. \\
\hline Yes & 1.03 & $\begin{array}{l}(0.97- \\
1.08)\end{array}$ & 1.14 & $\begin{array}{l}(1.08- \\
1.19)\end{array}$ & 0.99 & $\begin{array}{l}(0.90- \\
1.09)\end{array}$ & 1.04 & $\begin{array}{l}(0.99- \\
1.08)\end{array}$ \\
\hline \multicolumn{9}{|l|}{$\begin{array}{l}\text { PCa risk } \\
\text { category }\end{array}$} \\
\hline No PCa & 1.00 & ref. & 1.00 & ref. & 1.00 & ref. & 1.00 & ref. \\
\hline Low risk & 0.95 & $\begin{array}{l}(0.84- \\
1.06)\end{array}$ & 1.04 & $\begin{array}{l}(0.94- \\
1.15)\end{array}$ & 1.12 & $\begin{array}{l}(0.95- \\
1.32)\end{array}$ & 0.99 & $\begin{array}{l}(0.90- \\
1.08)\end{array}$ \\
\hline Intermediate risk & 1.01 & $\begin{array}{l}(0.92- \\
1.10)\end{array}$ & 1.05 & $\begin{array}{l}(0.97- \\
1.14)\end{array}$ & 0.95 & $\begin{array}{l}(0.81- \\
1.10)\end{array}$ & 1.02 & $\begin{array}{l}(0.95- \\
1.10)\end{array}$ \\
\hline High risk & 0.97 & $\begin{array}{l}(0.87- \\
1.07)\end{array}$ & 1.13 & $\begin{array}{l}(1.03- \\
1.24)\end{array}$ & 0.90 & $\begin{array}{l}(0.75- \\
1.07)\end{array}$ & 1.00 & $\begin{array}{l}(0.92- \\
1.08)\end{array}$ \\
\hline $\begin{array}{l}\text { Regional } \\
\text { metastases }\end{array}$ & 1.29 & $\begin{array}{l}(1.07- \\
1.56)\end{array}$ & 1.47 & $\begin{array}{l}(1.24- \\
1.74)\end{array}$ & 1.14 & $\begin{array}{l}(0.82- \\
1.59)\end{array}$ & 1.28 & $\begin{array}{l}(1.10- \\
1.50)\end{array}$ \\
\hline $\begin{array}{l}\text { Distance } \\
\text { metastases }\end{array}$ & 1.36 & $\begin{array}{l}(1.16- \\
1.60)\end{array}$ & 1.59 & $\begin{array}{l}(1.38- \\
1.82)\end{array}$ & 0.88 & $\begin{array}{l}(0.63- \\
1.22)\end{array}$ & 1.23 & $\begin{array}{l}(1.09- \\
1.40)\end{array}$ \\
\hline Missing data & 0.96 & $\begin{array}{l}(0.71- \\
1.29)\end{array}$ & 1.01 & $\begin{array}{l}(0.78- \\
1.31)\end{array}$ & 1.22 & $\begin{array}{l}(0.80- \\
1.88)\end{array}$ & 0.92 & $\begin{array}{l}(0.73- \\
1.16)\end{array}$ \\
\hline \multicolumn{9}{|l|}{$\begin{array}{l}\text { GnRH agonists, } \\
\text { n (\%) }\end{array}$} \\
\hline No PCa & 1.00 & ref. & 1.00 & ref. & 1.00 & ref. & 1.00 & ref. \\
\hline No & 0.97 & $\begin{array}{l}(0.91- \\
1.03)\end{array}$ & 1.06 & $(1.00-1.12)$ & 0.94 & $\begin{array}{l}(0.84- \\
1.04)\end{array}$ & 0.98 & $\begin{array}{l}(0.93- \\
1.03)\end{array}$ \\
\hline Yes & 1.23 & $\begin{array}{l}(1.11- \\
1.36)\end{array}$ & 1.41 & $\begin{array}{l}(1.28- \\
1.54)\end{array}$ & 1.20 & $\begin{array}{l}(1.01- \\
1.42)\end{array}$ & 1.24 & $\begin{array}{l}(1.13- \\
1.34)\end{array}$ \\
\hline \multicolumn{9}{|c|}{ a. Men with a $\mathrm{HbA} 1 \mathrm{c}$ over $58 \mathrm{mmol} / \mathrm{I}$ at baseline excluded } \\
\hline \multicolumn{9}{|c|}{ b. Men using insulin at the baseline excluded } \\
\hline
\end{tabular}


Men receiving $\mathrm{GnRH}$ agonists had a higher cumulative incidence for worsening diabetes control, compared to PCa free men (Fig. 2). The changes in the HbA1c measurements (Fig. 2-a, Fig. 2-b) occurred earlier than the addition of new antidiabetic medications (Fig. 2-c).

\section{$\mathrm{PCa}+\mathrm{GnRH}$ Cohort}

PCa men GnRH had a higher risk of worsening diabetes control compared to men with PCa not on GnRH agonists (HR:1.58, 95\% Cl: 1.39-1.80) (Table 3). No differences by PCa risk categories were observed (Table 3). The HR for worsening diabetes control was 1.34 (95\% Cl: $0.97-1.83$ ) in regional metastatic $\mathrm{PCa}$, and 1.08 (95\% Cl: 0.72-1.62) in men with distant metastases. 
Table 3

$\mathrm{HR}$ and $95 \% \mathrm{Cl}$ for change in diabetes control in $\mathrm{PCa}+\mathrm{GnRH}$ cohort

$\begin{array}{llll}\mathrm{HbA} 1 \mathrm{c} \text { equal to } & \mathrm{HbA} 1 \mathrm{c} \text { increased } & \begin{array}{l}\text { Change of } \\ \text { T2DM drugs }\end{array} & \begin{array}{l}\text { Combination of } \\ \text { all definitions }\end{array}\end{array}$

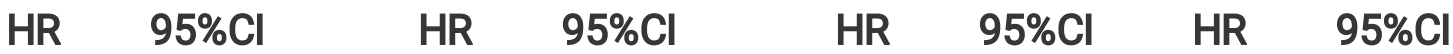

Crude model

Using GnRH

agonists, $\mathrm{n}(\%)$

\begin{tabular}{|c|c|c|c|c|c|c|c|}
\hline No & 1.00 & ref. & 1.00 & ref. & 1.00 & ref. & 1.00 \\
\hline Yes & 1.60 & $\begin{array}{l}(1.37- \\
1.87)\end{array}$ & 1.77 & $\begin{array}{l}(1.53- \\
2.04)\end{array}$ & 1.14 & $\begin{array}{l}(0.85- \\
1.53)\end{array}$ & 1.56 \\
\hline
\end{tabular}

PCa risk

category

\begin{tabular}{|c|c|c|c|c|c|c|c|c|}
\hline Low risk & 1.00 & ref. & 1.00 & ref. & 1.00 & ref. & 1.00 & ref. \\
\hline Intermediate risk & 1.12 & $\begin{array}{l}(0.98- \\
1.29)\end{array}$ & 1.00 & $\begin{array}{l}(0.87- \\
1.15)\end{array}$ & 0.97 & $\begin{array}{l}(0.76- \\
1.23)\end{array}$ & 0.99 & $\begin{array}{l}(0.88- \\
1.12)\end{array}$ \\
\hline High risk & 1.08 & $\begin{array}{l}(0.90- \\
1.29)\end{array}$ & 1.11 & $\begin{array}{l}(0.94- \\
1.30)\end{array}$ & 0.77 & $\begin{array}{l}(0.56- \\
1.07)\end{array}$ & 1.05 & $\begin{array}{l}(0.91- \\
1.21)\end{array}$ \\
\hline $\begin{array}{l}\text { Regional } \\
\text { metastases }\end{array}$ & 1.35 & $\begin{array}{l}(0.92- \\
1.97)\end{array}$ & 1.38 & $\begin{array}{l}(0.96- \\
1.98)\end{array}$ & 0.84 & $\begin{array}{l}(0.39- \\
1.80)\end{array}$ & 1.33 & $\begin{array}{l}(0.97- \\
1.82)\end{array}$ \\
\hline $\begin{array}{l}\text { Distance } \\
\text { metastases }\end{array}$ & 1.31 & $\begin{array}{l}(0.80- \\
2.13)\end{array}$ & 1.32 & $\begin{array}{l}(0.84- \\
2.06)\end{array}$ & 1.04 & $\begin{array}{l}(0.46- \\
2.36)\end{array}$ & 1.11 & $\begin{array}{l}(0.75- \\
1.65)\end{array}$ \\
\hline Missing data & 0.63 & $\begin{array}{l}(0.39- \\
1.02)\end{array}$ & 0.83 & $\begin{array}{l}(0.57- \\
1.22)\end{array}$ & 0.66 & $\begin{array}{l}(0.29- \\
1.49)\end{array}$ & 0.81 & $\begin{array}{l}(0.59- \\
1.12)\end{array}$ \\
\hline \multicolumn{9}{|l|}{$\begin{array}{l}\text { Adjusted model } \\
\text { c }\end{array}$} \\
\hline \multicolumn{9}{|l|}{$\begin{array}{l}\text { Using GnRH } \\
\text { agonists, } \mathrm{n}(\%)\end{array}$} \\
\hline No & 1.00 & ref. & 1.00 & ref. & 1.00 & ref. & 1.00 & ref. \\
\hline Yes & 1.56 & $\begin{array}{l}(1.33- \\
1.83)\end{array}$ & 1.78 & $\begin{array}{l}(1.54- \\
2.06)\end{array}$ & 1.21 & $\begin{array}{l}(0.89- \\
1.63)\end{array}$ & 1.58 & $\begin{array}{l}(1.39- \\
1.80)\end{array}$ \\
\hline
\end{tabular}

a. We excluded men with a $\mathrm{HbA} 1 \mathrm{c}$ over $58 \mathrm{mmol} / \mathrm{l}$ at baseline

b. We excluded men using insulin at the baseline

c. This model was adjusted for age at PCa diagnosis, duration of T2DM, education level, $\mathrm{CCl}$, civil status, smoking habits, physical activity and BMI 


\begin{tabular}{|c|c|c|c|c|c|c|c|c|}
\hline & \multicolumn{2}{|c|}{$\begin{array}{l}\text { HbA1c equal to } \\
58 \mathrm{mmol} / \mathrm{mol}^{\mathrm{a}}\end{array}$} & \multicolumn{2}{|c|}{$\begin{array}{l}\text { HbA1c increased } \\
10 \mathrm{mmol} / \mathrm{mol}\end{array}$} & \multicolumn{2}{|c|}{$\begin{array}{l}\text { Change of } \\
\text { T2DM drugs }\end{array}$} & \multicolumn{2}{|c|}{$\begin{array}{l}\text { Combination of } \\
\text { all definitions }\end{array}$} \\
\hline & HR & $95 \% \mathrm{Cl}$ & HR & $95 \% \mathrm{Cl}$ & HR & $95 \% \mathrm{Cl}$ & HR & $95 \% \mathrm{Cl}$ \\
\hline \multicolumn{9}{|l|}{$\begin{array}{l}\text { PCa risk } \\
\text { category }\end{array}$} \\
\hline Low risk & 1.00 & ref. & 1.00 & ref. & 1.00 & ref. & 1.00 & ref. \\
\hline Intermediate risk & 1.08 & $\begin{array}{l}(0.94- \\
1.26)\end{array}$ & 0.99 & $\begin{array}{l}(0.86- \\
1.13)\end{array}$ & 1.00 & $\begin{array}{l}(0.78- \\
1.28)\end{array}$ & 0.99 & $\begin{array}{l}(0.88- \\
1.11)\end{array}$ \\
\hline High risk & 0.99 & $\begin{array}{l}(0.83- \\
1.20)\end{array}$ & 1.06 & $\begin{array}{l}(0.90- \\
1.26)\end{array}$ & 0.83 & $\begin{array}{l}(0.60- \\
1.16)\end{array}$ & 1.02 & $\begin{array}{l}(0.88- \\
1.18)\end{array}$ \\
\hline $\begin{array}{l}\text { Regional } \\
\text { metastasises }\end{array}$ & 1.28 & $\begin{array}{l}(0.87- \\
1.87)\end{array}$ & 1.38 & $\begin{array}{l}(0.96- \\
1.98)\end{array}$ & 0.86 & $\begin{array}{l}(0.40- \\
1.85)\end{array}$ & 1.34 & $\begin{array}{l}(0.97- \\
1.83)\end{array}$ \\
\hline $\begin{array}{l}\text { Distance } \\
\text { metastasises }\end{array}$ & 1.25 & $\begin{array}{l}(0.77- \\
2.04)\end{array}$ & 1.25 & $\begin{array}{l}(0.80- \\
1.97)\end{array}$ & 1.12 & $\begin{array}{l}(0.49- \\
2.55)\end{array}$ & 1.08 & $\begin{array}{l}(0.72- \\
1.62)\end{array}$ \\
\hline Missing data & 0.56 & $\begin{array}{l}(0.34- \\
0.92)\end{array}$ & 0.81 & $\begin{array}{l}(0.55- \\
1.19)\end{array}$ & 0.63 & $\begin{array}{l}(0.27- \\
1.43)\end{array}$ & 0.78 & $\begin{array}{l}(0.57- \\
1.09)\end{array}$ \\
\hline \multicolumn{9}{|c|}{ a. We excluded men with a $\mathrm{HbA} 1 \mathrm{c}$ over $58 \mathrm{mmol} / \mathrm{l}$ at baseline } \\
\hline \multicolumn{9}{|c|}{ b. We excluded men using insulin at the baseline } \\
\hline $\begin{array}{l}\text { c. This model wo } \\
\text { status, smoking }\end{array}$ & just & $\begin{array}{l}\text { for age } \\
\text { ical ac }\end{array}$ & diag & sis, durati & T2D & educa & vel. & , civil \\
\hline
\end{tabular}

Men on GnRH had a worsening diabetes control compared to men with PCa not on GnRH over time (Fig. 3). The changes in HbA1c measurements (Fig. 3-a, Fig. 3-b) occurred earlier than the addition of new antidiabetic medications (Fig. 3-C), similar to that seen in the PCa cohort.

\section{Discussion}

In this nationwide, population-based study, use of GnRH was associated with worsening diabetes control in men with diabetes and PCa treated with GnRH.

\section{PCa cohort}

We found no statically significant association of PCa diagnosis (all risk categories) with worsening diabetes control in line with results of two previous studies $[3,16]$, which investigated the effect of PCa diagnosis on glycemic control and T2DM treatments.

Advanced $\mathrm{PCa}$, including regional metastatic and distance metastatic disease, was associated with worsening of diabetes control compared to men without PCa [3]. This finding may be explained by the use of GnRH in men with advanced PCa [4]. GnRH reduce insulin sensitivity [17], which could lead to 
worsening of diabetic control [18]. Therefore, their use may explain the association of worsening T2DM control with more advanced PCa.

In addition, starting GnRH worsened diabetes control in T2DM men compared to men with diabetes without PCa in accordance with results of previous observational studies $[3,19]$. These showed that men with PCa treated with GnRH agonists had an increased risk of T2DM treatment escalations, compared to men with PCa not on $\mathrm{GnRH}$ agonists [3] and use of $\mathrm{GnRH}$ increased $\mathrm{HbA1c}$ level and worsened diabetes control in men with PCa [19].

\section{$\mathrm{PCa}+\mathrm{GnRH}$ cohort}

Next, we wanted to demonstrate that the association with worsening diabetes control was caused by the $\mathrm{GnRH}$ rather than the PCa diagnosis per se. In the PCa $+\mathrm{GnRH}$ cohort T2DM men with PCa who started $\mathrm{GnRH}$ after PCa diagnosis had worse diabetes control than men with PCa not on $\mathrm{GnRH}$, supporting the hypothesis that it is the GnRH driving the worse control.

Several biological mechanisms have been proposed to explain the association between use of $\mathrm{GnRH}$ and worsening diabetes control[8]. Low levels of testosterone, induced by $\mathrm{GnRH}$, are implicated in the development of insulin resistance [20,21], which results in increased plasma glucose levels [22] and hence leads to worsening control of T2DM.

We found that changes in $\mathrm{HbA} 1 \mathrm{c}$ occurred prior to changes in T2DM drug use, which is logical since antidiabetic treatment will only be changed when the deterioration of diabetes control has been verified on repeat measures.

\section{Strengths and limitations}

Our study has several strengths. First, we were able to assemble a nationwide population-based cohort of men with T2DM from the largest diabetes register in the world, with up to 10 years of follow up. To our knowledge, this is the largest population-based cohort study exploring the association of use of $\mathrm{GnRH}$ agonists with diabetes control in men with T2DM. We were also able to disentangle the impact of a PCa diagnosis itself and explore the association with different risk categories of PCa. This was achieved by selecting men with T2DM with or without PCa and also with or without GnRH separately at baseline, thereby assembling two cohorts. Secondly, we were able to look at three separate markers of worsening glycaemic control due to the detailed longitudinal information within the NDR. Third, cases and relevant comparisons were matched on average time between two NDR visits. It reduced the impact of different time between visits, which is likely to be associated with quality of T2DM management. Finally, the NDR and PCBaSe 4.1 contain information on various critical confounders, including age, comorbidity, civil status, BMI, physical activity, and smoking status, which can be adjusted in the statistic models.

Limitations include that there was not sufficient power to fully explore the association of different risk categories of PCa on the worsening of T2DM control. Secondly, approximately $3-6 \%$ of men had missing data at baseline measurements. We used the last observation carried forward to impute the missing data, 
which may underestimate the effect of exposures. Last, residual confounding cannot be excluded, for example, by family history of T2DM and PCa.

\section{Conclusions}

In this large population-based cohort study, starting GnRH was associated with worsening of diabetes control in men with T2DM and PCa on GnRH agonists compared to matched PCa-free men with T2DM, as well as compared to men with T2DM and PCa not on $\mathrm{GnRH}$. Our findings highlight the need to closely monitor diabetes control in T2DM men with PCa and start of GnRH agonists and to limit the duration of their use when possible.

\section{List Of Abbreviations}

prostate cancer ( $\mathrm{PCa}$ ); Gonadotropin Releasing Hormones agonists ( $\mathrm{GnRH}$ ); type 2 diabetes mellitus (T2DM); Prostate Cancer data Base Sweden (PCBaSe); the National Prostate Cancer Register (NPCR); National Prescribed Drug Register (PDR); National Diabetes Registry (NDR); National Comprehensive Cancer Network (NCCN); Hazard ratios (HR); confidence interval (Cl); Charlson Comorbidity Index (CCl).

\section{Declarations}

\section{Ethics approval and consent to participate}

The study has been approved by The Research Ethics Board at Uppsala University, Sweden. As data of the study were obtained from the established national databases in Sweden, consent to participate and the experiment protocol were not required.

\section{Consent for publication}

Not applicable.

\section{Availability of data and materials}

The data that support the findings of this study are available from PCBaSe Sweden, but restrictions apply to the availability of these data, which were used under license for the current study, and so are not publicly available. Data are however available from the authors upon reasonable request and with permission of PCBaSe Sweden.

\section{Competing interests}

Hans Garmo, Mieke Van Hemelrijck, Jan Adolfsson, Pär Stattin and Björn Zethelius do not have competing interests.

\section{Funding}


This work was supported by The Swedish Research Council 2017-00847, The Swedish Cancer Society 16 0700, The Stockholm Cancer Society and The Henry Lester Trust. The funding bodies played no role in the study design, the data collection, the analysis, and the interpretation of data. They also did not involve in writing the manuscript.

\section{Authors' contributions}

E Lin, Danielle Crawley, Hans Garmo, Meike Van Hemelrijck and Björn Zethelius designed and coordinated this research. Pär Stattin and Hans Garmo created and administered the research database PCBaSe, including the linkages to the Diabetes Register. E Lin carried out the data with Hans Garmo, analyzed the data and was the major contributor in writing the manuscript. Hans Garmo, Jan Adolfsson, Pär Stattin and Björn Zethelius participated in research coordination. All authors read and approved the final manuscript.

\section{Acknowledgement}

This project was made possible by the continuous work of the National Prostate Cancer Register of Sweden (the National Prostate Cancer Register) steering group: Pär Stattin (chair), Ingela Franck Lissbrant (deputy chair), Johan Styrke, Camilla Thellenberg Karlsson, Lennart Åström, Stefan Carlsson, Marie Hjälm-Eriksson, David Robinson, Mats Andén, Ola Bratt, Magnus Törnblom, Jonas Hugosson, Maria Nyberg, Olof Akre, Per Fransson, Eva Johansson, Gert Malmberg, Fredrik Sandin, and Karin Hellström.

We thank Anne-Marie Svensson at the National Diabetes Register for coordination in linking NDR with PCBaSe.

\section{Disclaimer}

Björn Zethelius is employed at the Swedish Medical Products Agency, SE-751 03 Uppsala, Sweden. The views expressed in this paper are the personal views of the authors and not necessarily the views of the Swedish government agency.

\section{References}

1. Rawla P: Epidemiology of prostate cancer. World journal of oncology 2019, 10(2):63.

2. Eurepe Diabetes report 2010-2045

3. Crawley D, Garmo H, Rudman S, Stattin P, Zethelius B, Armes J, Holmberg L, Adolfsson J, Van Hemelrijck M: Does a prostate cancer diagnosis affect management of preexisting diabetes? Results from PCBaSe Sweden: a nationwide cohort study. BMJ open 2018, 8(3):e020787.

4. Rosario DJ, Davey P, Green J, Greene D, Turner B, Payne H, Kirby M: The role of gonadotrophinreleasing hormone antagonists in the treatment of patients with advanced hormone-dependent prostate cancer in the UK. World journal of urology 2016, 34(12):1601-1609. 
5. Zitzmann M: Testosterone deficiency, insulin resistance and the metabolic syndrome. Nature Reviews Endocrinology 2009, 5(12):673.

6. Østergren PB, Kistorp C, Fode M, Bennedbæk FN, Faber J, Sønksen J: Metabolic consequences of gonadotropin-releasing hormone agonists vs orchiectomy: a randomized clinical study. BJU international 2019, 123(4):602-611.

7. Huang G, Basaria S: Androgen deprivation therapy for prostate cancer: effects on body composition and metabolic health. In: Energy Balance and Prostate Cancer. edn.: Springer; 2018: 127-142.

8. Jhan J-H, Yeh H-C, Chang Y-H, Guu S-J, Wu W-J, Chou Y-H, Li C-C: New-onset diabetes after androgen-deprivation therapy for prostate cancer: A nationwide propensity score-matched four-year longitudinal cohort study. Journal of Diabetes and its Complications 2018, 32(7):688-692.

9. Saylor PJ, Keating NL, Freedland SJ, Smith MR: Gonadotropin-releasing hormone agonists and the risks of type 2 diabetes and cardiovascular disease in men with prostate cancer. In.: Springer; 2011.

10. Van Hemelrijck M, Wigertz A, Sandin F, Garmo H, Hellström K, Fransson P, Widmark A, Lambe M, Adolfsson J, Varenhorst E: Cohort profile: the national prostate cancer register of Sweden and prostate cancer data base Sweden 2.0. International journal of epidemiology 2013, 42(4):956-967.

11. Van Hemelrijck M, Garmo H, Wigertz A, Nilsson P, Stattin P: Cohort Profile Update: The National Prostate Cancer Register of Sweden and Prostate Cancer data Base-a refined prostate cancer trajectory. International journal of epidemiology 2016, 45(1):73-82.

12. Gudbjörnsdottir S, Cederholm J, Nilsson PM, Eliasson B: The National Diabetes Register in Sweden: an implementation of the St. Vincent declaration for quality improvement in diabetes care. Diabetes care 2003, 26(4):1270-1276.

13. Cederholm J, Eeg-Olofsson K, Eliasson B, Zethelius B, Nilsson PM, Gudbjörnsdottir S: Risk prediction of cardiovascular disease in type 2 diabetes: a risk equation from the Swedish National Diabetes Register. Diabetes care 2008, 31(10):2038-2043.

14. Mohler JL, Antonarakis ES, Armstrong AJ, D'Amico AV, Davis BJ, Dorff T, Eastham JA, Enke CA, Farrington TA, Higano CS et al: Prostate Cancer, Version 2.2019, NCCN Clinical Practice Guidelines in Oncology. Journal of the National Comprehensive Cancer Network: JNCCN2019, 17(5):479-505.

15. Excellence NIfC: Algorithm for blood glucose lowering therapy in adults with type 2 diabetes. In.; 2015.

16. Karlin NJ, Amin SB, Verona PM, Kosiorek HE, Cook CB: Co-existing prostate cancer and diabetes mellitus: implications for patient outcomes and care. Endocrine Practice 2017, 23(7):816-821.

17. Saylor PJ, Smith MR: Adverse effects of androgen deprivation therapy: defining the problem and promoting health among men with prostate cancer. Journal of the National Comprehensive Cancer Network 2010, 8(2):211-223.

18. Bradley MC, Zhou Y, Freedman AN, Yood MU, Quesenbery CP, Haque R, Van Den Eeden SK, CassidyBushrow AE, Aaronson D, Potosky AL: Risk of diabetes complications among those with diabetes receiving androgen deprivation therapy for localized prostate cancer. Cancer Causes \& Control 2018, 29(8):785-791. 
19. Keating NL, Liu P-H, O'Malley AJ, Freedland SJ, Smith MR: Androgen-deprivation therapy and diabetes control among diabetic men with prostate cancer. European urology 2014, 65(4):816-824.

20. Navarro G, Allard C, Xu W, Mauvais-Jarvis F: The role of androgens in metabolism, obesity, and diabetes in males and females. Obesity 2015, 23(4):713-719.

21. Yu I-C, Lin H-Y, Sparks JD, Yeh S, Chang C: Androgen receptor roles in insulin resistance and obesity in males: the linkage of androgen-deprivation therapy to metabolic syndrome. Diabetes 2014, 63(10):3180-3188.

22. Taylor R: Insulin resistance and type 2 diabetes. Diabetes 2012, 61(4):778-779.

\section{Figures}




\section{NDR in PCBaSe4.1}

2941,720 registrations 231,334 men
Exclude T1DM

235,270 registrations

27,118 men

Exclude registrations prior to 1/1 2006

146,670 registrations

2,223 men

Exclude men in PCBaSe4.1 that are neither registered in NPCR nor randomly selected as comparison men

843,445 registrations

52,483 men
T2DM registered in NDR from

1/1 2006

$1,716,335$ registrations 149,510 men

Exclude men with four or fewer registrations in NDR

96,486 registrations

40,118 men

Eligible to study

$1,520,047$ registrations

101,843 men

Exclude men 1) diagnosed with PCa at baseline in PCa cohort, and 2) men used GnRH at baseline in PCa+GnRH cohort 99,802 registrations

7,485 men

Study of exposure change

PCa vs. No PCa (PCa cohort)

5,714 cases

28,445 comparison men 
with PCa and 28,445 PCa-free men were included in the PCa cohort. The PCa+GnRH cohort contained 692 PCa men who started GnRH after PCa diagnosis and 3,460 PCa men not using GnRH as comparison.

$$
\text { - NoPCa - PCa } \quad-\mathrm{PCa}+\mathrm{GnRH}
$$
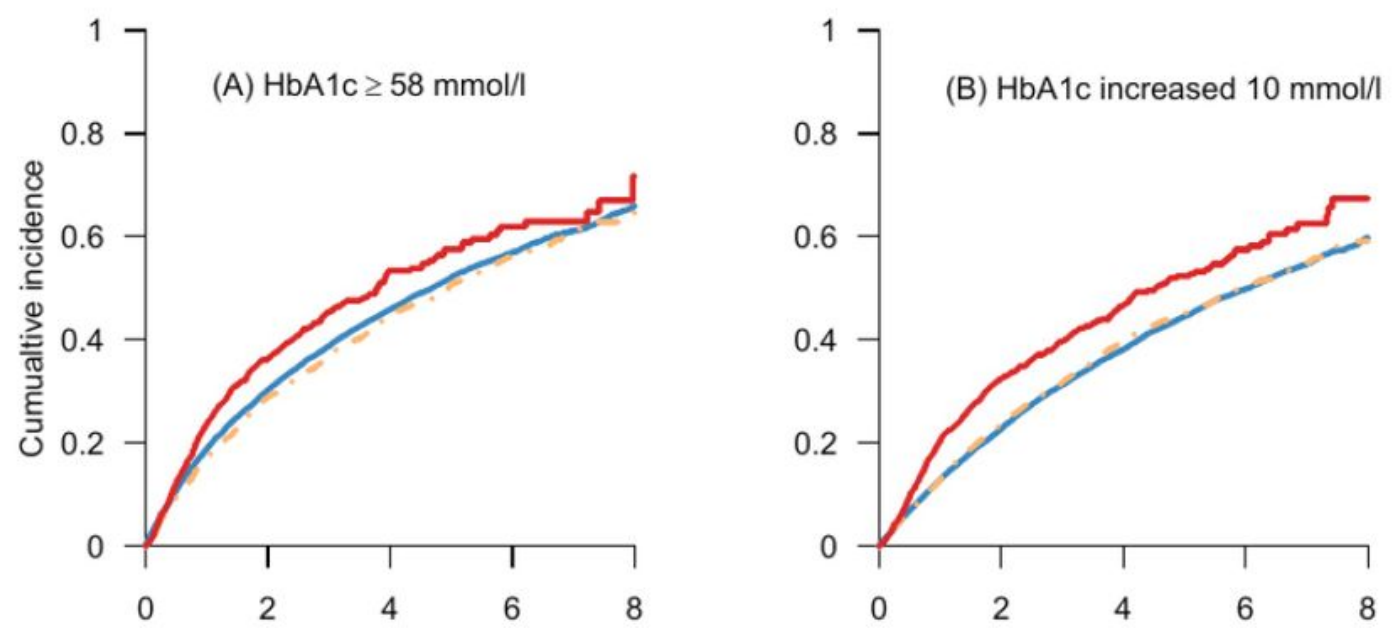

Number at risk
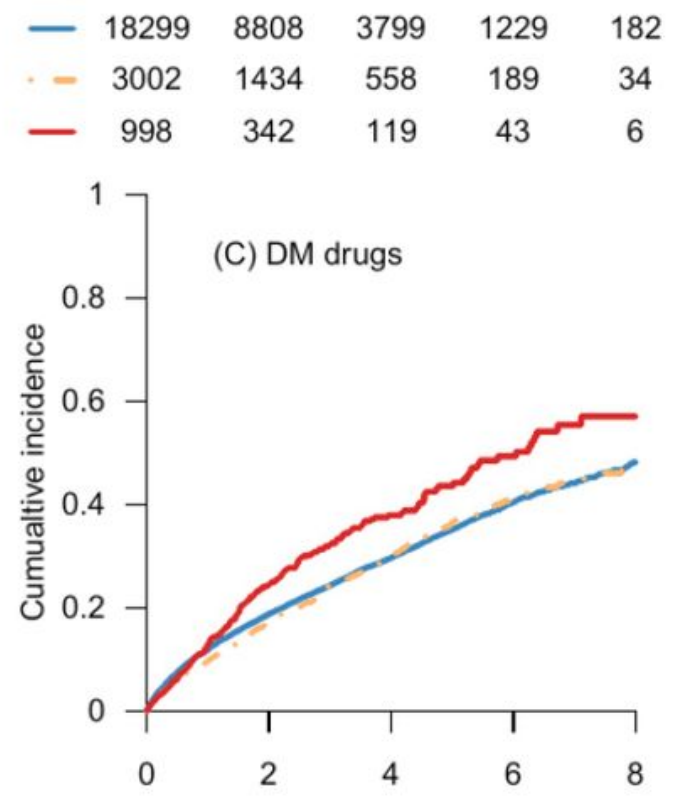

Number at risk

\begin{tabular}{|c|c|c|c|c|}
\hline 18186 & 10075 & 4804 & 1595 & 276 \\
\hline 2905 & 1612 & 705 & 247 & 47 \\
\hline 936 & 385 & 149 & 59 & 11 \\
\hline
\end{tabular}

Number at risk

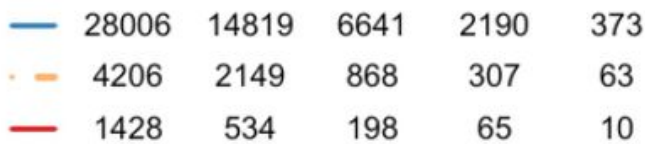

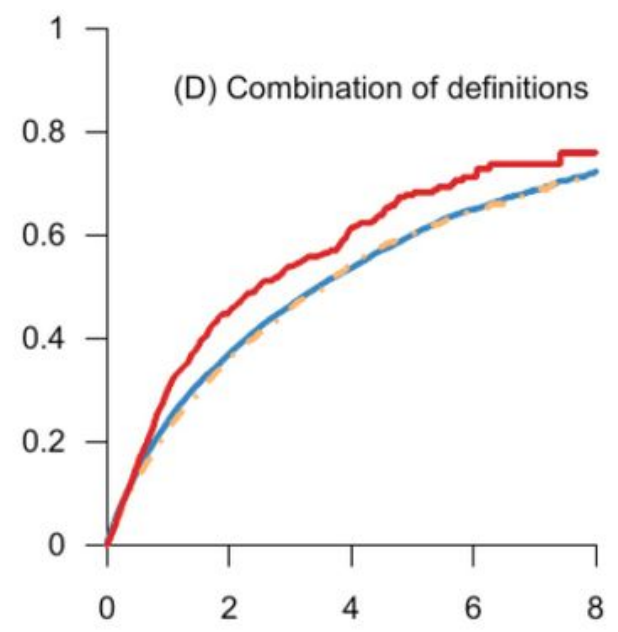

Number at risk

\begin{tabular}{ccccc}
-28247 & 12103 & 4925 & 1482 & 227 \\
$-\quad 4251$ & 1807 & 638 & 209 & 43 \\
-1435 & 421 & 129 & 38 & 6 \\
& \multicolumn{4}{c}{ Time to event (years) }
\end{tabular}

\section{Figure 2}

Cumulative incidence of worsening T2DM control in T2DM men by PCa status in PCa Cohort In this figure, we found that, in the PCa cohort, men receiving $\mathrm{GnRH}$ agonists had a higher cumulative incidence for worsening diabetes control, compared to PCa free men. The changes in the HbA1c measurements 
(Figure 2-(a), Figure 2-(b)) occurred earlier and more obviously than the addition of new antidiabetic medications (Figure 2-(c)). When we combined the criteria to identify the event in Figure 2-(a), Figure 2-(b) and Figure 2-(c) to create the combination event, we found that cumulative incidence of combination event is higher in PCa men with GnRH compared with men without PCa (Figure 2-(d)).
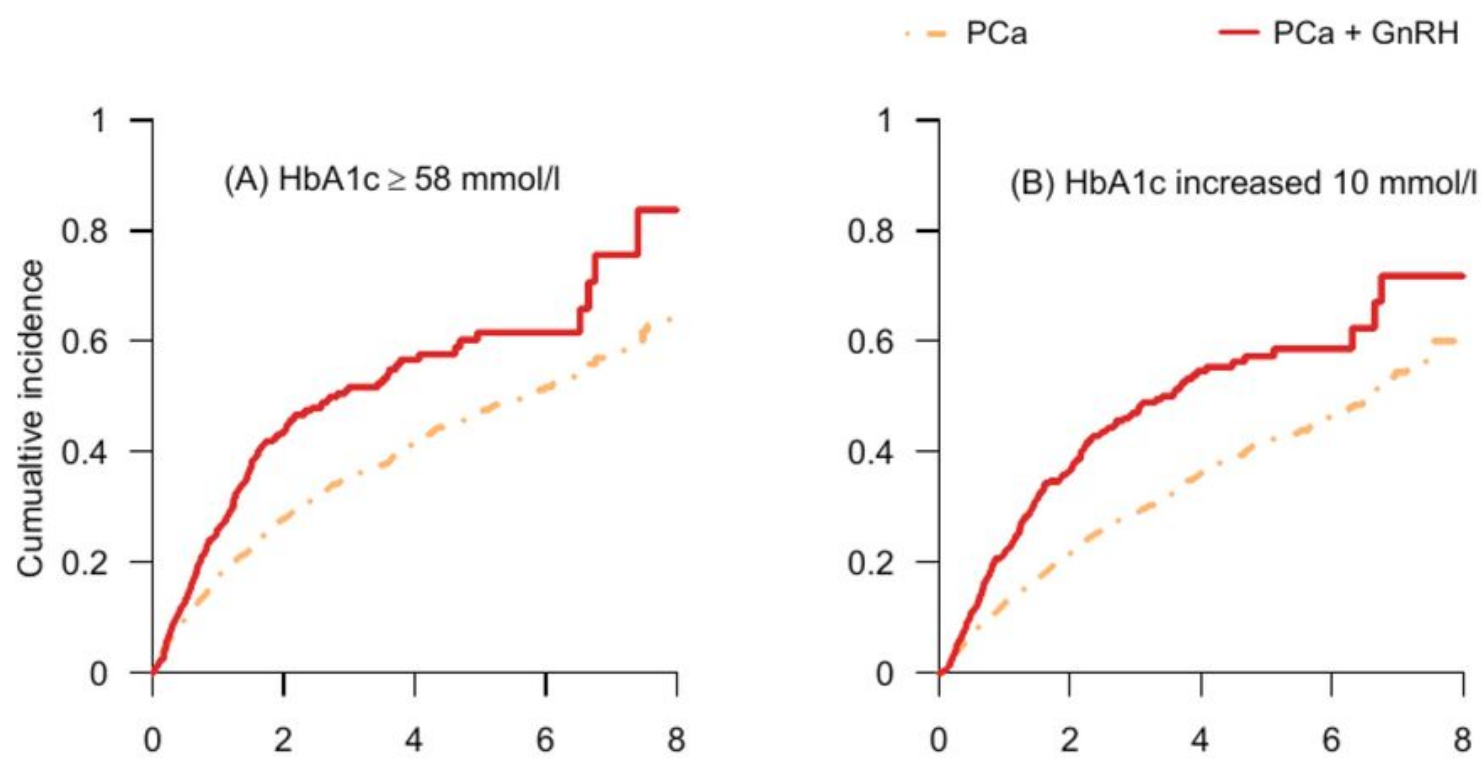

Number at risk

Number at risk

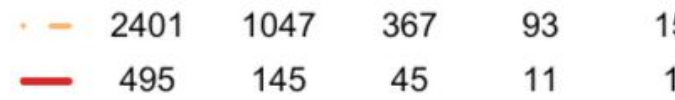

\begin{tabular}{|c|c|c|c|}
\hline 3386 & 1559 & 572 & 143 \\
\hline 677 & 209 & 65 & 13 \\
\hline
\end{tabular}
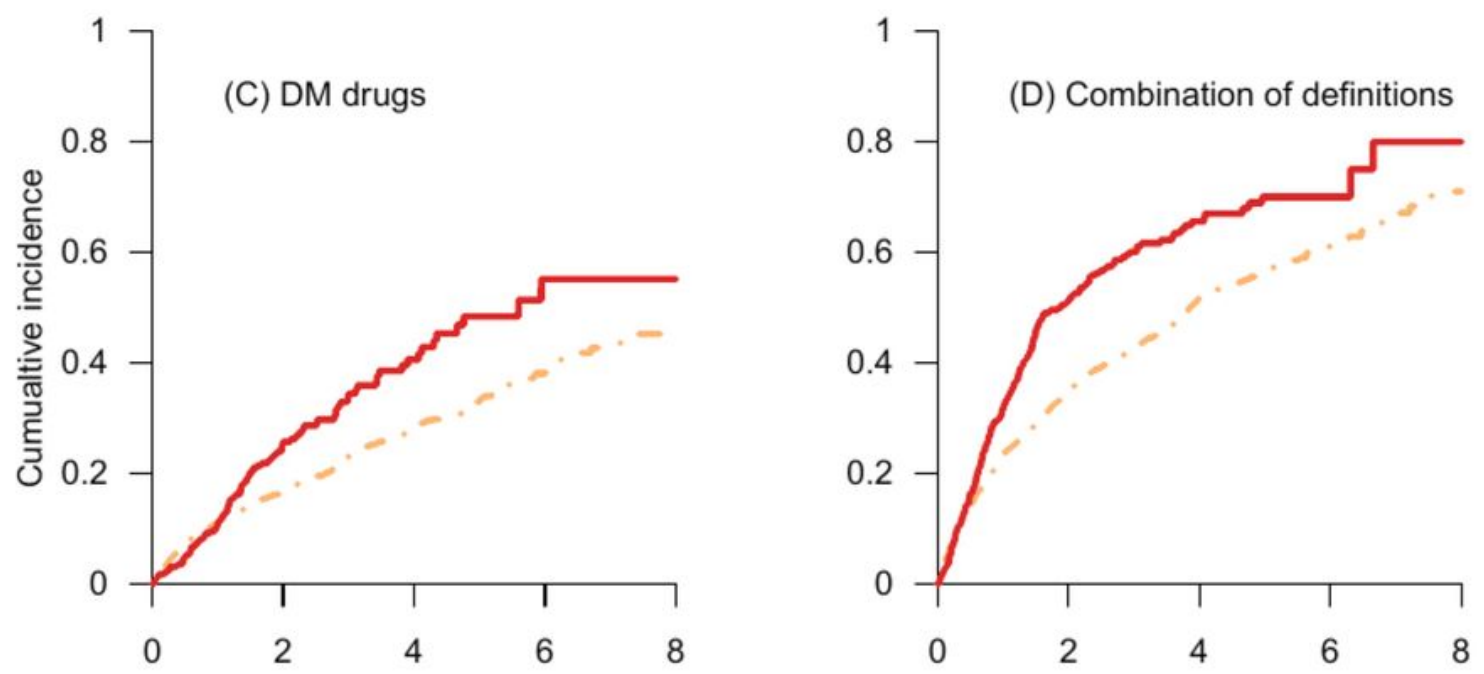

Number at risk

Number at risk

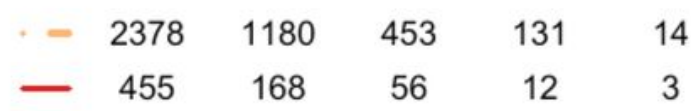

Time to event (years)

Time to event (years)

Figure 3 
Cumulative incidence of worsening T2DM control in T2DM men by using GnRH in PCa+GnRH cohort showed Kaplan Meier Curves for cumulative incidence of worsening T2DM control in T2DM men in $\mathrm{PCa}+\mathrm{GnRH}$ cohort. It presented those men on $\mathrm{GnRH}$ had a worsening diabetes control compared to men with PCa not on GnRH over time. The changes in HbA1c measurements (Figure 3-(a), Figure 3-(b)) occurred earlier and more obviously than the addition of new antidiabetic medications (Figure 3-(c)). Figure 3-(d) showed the cumulative incidence of combination of definitions which was combined by criteria of worsening diabetes control in Figure 3-(a), Figure 3-(b) and Figure 3-(c). We observed that cumulative incidence of combination of definitions is higher in PCa men with GnRH compared with PCa men but not on GnRH over time. 